\title{
Echo-enhanced needles for short-axis ultrasound-guided vascular access
}

\author{
Kumaresh Venkatesan
}

Received: 31 December 2009 / Accepted: 18 January 2010/Published online: 10 April 2010

(C) Springer-Verlag London Ltd 2010

Dear Editors,

I read with interest the study by Phelan et al. [1]. The authors should be commended for their study evaluating the use of echo-enhanced needles in obtaining ultrasoundguided vascular access using a short-axis view.

Historically, ultrasound has been used to guide needle, catheter and guidewire placement by radiologists but it now is being used increasingly by anaesthesiologists, emergency and critical care physicians for vascular access, nerve plexus blockade and drainage of pleural or ascitic fluid collections. Ultrasound allows identification of the target and collateral vascular structures and real-time guidance to precisely place needles. As the authors state, traditionally the short-axis view is used for vascular access as it allows the visualisation of the vein and accompanying artery in one image. Needle visualisation, i.e. the ability to assess the position of the advancing needle tip, is crucial to the success and safety of the technique. Commercially available echo-enhanced needles have been in vogue in radiology practice for some time and have the theoretical advantage of the better visualisation compared with a standard needle.

The study by Phelan et al. is probably the first study evaluating the usefulness of echo-enhanced needles in performing real-time ultrasound-guided vascular access, albeit in a vascular access model. The authors have concluded that there was no difference in the procedure performance times or the needle manipulations. I wish to raise a few issues regarding the study methodology. The

K. Venkatesan $(\bowtie)$

New Cross Hospital,

Wolverhampton WV10 0QP, UK

e-mail: kumareshvenkatesan@doctors.org.uk authors state that the aim of the study was to determine if the use of an echo-enhanced needle tip "resulted in faster vascular access times, with fewer needle sticks, fewer redirections, and improved needle visualization in ultrasound-guided vascular access with the vessel in the short axis". The perceived advantage of an echoenhanced needle is superior visibility compared to standard needles. Theoretically, improved needle visualisation should translate into faster procedure performance times or needle manipulations. However, the authors have not objectively assessed whether use of the echoenhanced needle tip resulted in superior needle visibility compared to standard needle tips. Objective tools to assess needle visibility are available and have been described in previous studies [2, 3]. Therefore the authors' conclusion that use of an echo-enhanced needle tip did not improve procedure performance times or needle manipulations is perhaps overstated.

The differences in the visibility of the standard and echoenhanced vascular access needles in tissue phantom models and human tissues and their effect on performance, success and safety needs further formal evaluation.

\section{References}

1. Phelan MP, Emerman C, Peacock WF et al (2009) Do echoenhanced needles improve time to cannulate in a model of shortaxis ultrasound-guided vascular access for a group of mostly inexperienced ultrasound users? Int J Emerg Med 2:167-170

2. Schafhalter-Zoppoth I, McCulloch CE, Gray AT (2004) Ultrasound visibility of needles used for regional nerve block: an in vitro study. Reg Anesth Pain Med 29(5):480-488

3. Hopkins RE, Bradley M (2001) In-vitro visualization of biopsy needles with ultrasound: a comparative study of standard and echogenic needles using an ultrasound phantom. Clin Radiol 56 (6):499-502 